\title{
What do Galactic electrons and positrons tell us about dark matter?
}

\author{
Mathieu Boudaud ${ }^{* a}$ and E. F. Bueno ${ }^{b}$, S. Caroff ${ }^{c}$, A. Putze ${ }^{d}$, Y. Genolini $^{d}$, J. Lavalle ${ }^{e}$, \\ V. Poireau ${ }^{c}$, V. Poulin ${ }^{d}$, S. Rosier ${ }^{c}$, P. Salati ${ }^{d}$, M. Vecchi ${ }^{b}$ \\ ${ }^{a}$ LPTHE, CNRS \& UPMC, 4 Place Jussieu, F-75252 Paris - France

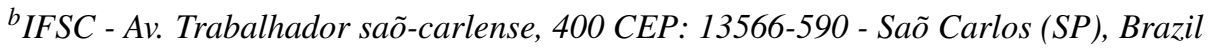 \\ ${ }^{c}$ LAPP, USMB \& CNRS, 9 Chemin de Bellevue, F-74941, Annecy-le-Vieux, France \\ ${ }^{d}$ LAPTh, USMB \& CNRS, 9 Chemin de Bellevue, F-74941, Annecy-le-Vieux, France \\ ${ }^{e}$ LUPM, UM \& CNRS, Place Eugène Bataillon, F-34095, Montpellier, France
}

E-mail: boudaudelpthe.jussieu. fr

\begin{abstract}
We devise a new semi-analytical method dedicated to the propagation of Galactic electrons and positrons from $\mathrm{MeV}$ to $\mathrm{TeV}$ energies: the pinching method. It is essentially based on the pinching of inverse Compton and synchrotron energy losses from the magnetic halo, where they take place, inside the Galactic disc. This new tool is fast and allows to carry out extensive scans over parameters. We strongly constrain the cosmic ray propagation parameters by requiring that the secondary component of positrons does not overshoot the AMS-02 measurements. We find that only models with a large diffusion coefficient and a large magnetic halo size are selected by this test. Therefore, we find that the positron excess appears from $1 \mathrm{GeV}$. We then explore the possibility to explain the positron excess with a component coming from the annihilation of dark matter particles. We show that the pure dark matter interpretation of the AMS-02 positron data is strongly disfavoured. This conclusion is based solely on the positron data, and no other observation, such as the antiproton and gamma ray fluxes or the CMB anisotropies, needs to be invoked. $\mathrm{MeV}$ dark matter particles annihilating or decaying to electron-positron pairs cannot, in principle, be observed via local cosmic ray measurements because of the shielding solar magnetic field. We take advantage of spacecraft Voyager-I's capacity for detecting interstellar cosmic rays since it crossed the heliopause in 2012. This opens up a new avenue to probe dark matter particles in the sub-GeV energy/mass range that we exploit here for the first time.
\end{abstract}

35th International Cosmic Ray Conference - ICRC2017

10-20 July, 2017

Bexco, Busan, Korea

* Speaker. 


\section{Introduction}

The cosmic ray (CR) positron flux has been measured with unprecedented accuracy by the AMS-02 collaboration [1]. The data confirm the positron excess: an additional component on top of the secondary one originating from the spallation of the gaz lying in the Galactic disc is required. The vast majority of these studies are focused on the high-energy part of the positron spectrum, namely above $10 \mathrm{GeV}$. Below this energy, Galactic convection, diffusive reacceleration, and positron annihilation on interstellar gaz must be taken into account. In addition, energy losses, which takes a key role in the propagation of positrons, are mostly concentrated in the Galactic disc whereas they extend all over the magnetic halo at high energy. Finally, solar modulation comes into play and complicates the interpretation of the data. Of course, looking for a dark matter (DM) solution of the positron anomaly by fitting the DM particle annihilating cross-section and mass to the high-energy part of the data is tantalising (see for example [2]). But these analyses are based on the prejudice that low-energy positron data are not relevant to DM, an unwarranted assumption that might introduce biases. These considerations have led us to reinvestigate the problem of the positron anomaly over the entire energy range covered by the AMS-02 data. To do so, we have conceived a new semi-analytical method to cope with transport processes so far neglected but important below a few $\mathrm{GeV}$ : the pinching method. An essential benefit of this tool is a faster runtime in comparison to a fully numerical approach, allowing to carry out extensive scans over the CR propagation parameters.

Below, we present the pinching method and the implications for the positron flux of CR transport processes so far neglected at high energies [4]. Then, we constrain the CR propagation parameters requiring that they do not lead to a flux of secondary positrons in excess of the measurements. The DM interpretation of the positron flux reported by AMS-02 is investigated. Finally, we took advantage of this method to derive for the first time constraints on MeV DM from the Voyager-I data [9].

\section{The pinching method}

Under a steady state and thin disc approximation, the density of CRs per unit of space and energy $\psi(E, r, z) \equiv d N / d^{3} x d E$ obeys the transport equation

$$
\nabla \cdot\left[V_{c} \psi-K(E) \nabla \psi\right]+\partial_{E}\left[b(E, z) \psi-2 h \delta(z) D(E) \partial_{E} \psi\right]+2 h \delta(z) \Gamma \psi=Q(E, r, z),
$$

where $V_{c}$ is the Galactic wind velocity, $K$ is the isotropic and homogeneous spatial diffusion coefficient, $D$ stands for the diffusive reacceleration and $b$ and $\Gamma$ are the energy losses and annihilation rates, respectively. In the r.h.s, $Q$ represents the injection rate of CRs in the Galaxy. We split the energy losses $b(E, z)$ into a disc component $b_{\text {disc }} \equiv b_{\text {coul }}+b_{\text {brem }}+b_{\text {ioni }}+b_{\text {adia }}$ that includes the mechanisms that take place only in the Galactic disc, and a halo component $b_{\text {halo }} \equiv b_{\mathrm{IC}}+b_{\text {sync }}$ considering those that take place in the whole magnetic halo (including the disc). CR nuclei lose energy only in the Galactic disc (i.e. $b_{\text {halo }}^{\text {nuc }} \simeq 0$ ). In this case, the transport equation (2.1) can be solved via the semi-analytical scheme introduced in [3]. More precisely, the CR density $\psi$ is expanded on the 
basis of the first-order Bessel functions $J_{0}$ such that

$$
\psi(E, r, z)=\sum_{i=1}^{\infty} J_{0}\left(\alpha_{i} \frac{r}{R}\right) P_{i}(E, z)
$$

where $\alpha_{i}$ are the zero of the Bessel function $J_{0}$ and $R$ the radius of the Galaxy. Therefore, eq 2.1 can be solved numerically using a Cranck-Nicholson algorithm. At first sight, it seems that the semianalytical method cannot be used to solve Eq. (2.1) when energy losses take place simultaneously in the magnetic halo and in the Galactic disc. Indeed, the difficulty comes from the fact that electrons lose energy in the Galactic disc as well as in the whole magnetic halo. The presence of the term $b_{\text {halo }}$ prevents a direct semi-analytical resolution. The trick to overcome this issue is to impose the halo energy losses to take place, in an effective way, only in the Galactic disc. In other words, it consists in replacing the term $b_{\text {halo }}$ in the transport equation (2.1) with an effective term $2 h \delta(z) b_{\text {halo }}^{\text {eff }}$ while keeping the same solution $\psi$. This condition enables us to determine the function $b_{\text {halo }}^{\text {eff }}$ defined as $b_{\text {halo }}^{\text {eff }}(E, r)=\bar{\xi}(E, r) b_{\text {halo }}(E)$. The pinching factor $\bar{\xi}$ is then given by

$$
\bar{\xi}(E, r)=\frac{1}{\psi^{h}(E, r, 0)} \sum_{i=1}^{+\infty} J_{0}\left(\alpha_{i} \frac{r}{R}\right) \bar{\xi}_{i}(E) P_{i}(E, 0),
$$

where

$$
\begin{gathered}
P_{i}(E, 0)=\frac{-1}{b_{\text {halo }}(E)} \int_{E}^{+\infty} d E_{S} B_{i}\left(E, E_{S}\right), \\
B_{i}\left(E, E_{S}\right)=\sum_{n=2 m+1}^{+\infty} Q_{i, n}\left(E_{S}\right) \exp \left[-C_{i, n} \lambda_{D}^{2}\right],
\end{gathered}
$$

and $\psi^{h}$ is solution of 2.1 in the high energy approximation (only halo energy losses and diffusion). The function $Q_{i, n}$ is the Bessel and Fourrier transform of $Q(E, z)$,

$$
C_{i, n}=\frac{1}{4}\left[\left(\frac{\alpha_{i}}{R}\right)^{2}+\left(n k_{0}\right)^{2}\right], \quad k_{0}=\pi / 2 L,
$$

and the diffusion length $\lambda_{D}$ is related to the space diffusion coefficient $K$ and the energy loss rate $b_{\text {halo }}$ by the expression

$$
\lambda_{D}^{2}\left(E, E_{S}\right)=4 \int_{E_{S}}^{E} d E^{\prime} \frac{K\left(E^{\prime}\right)}{b_{\text {halo }}\left(E^{\prime}\right)} .
$$

Finally,

$$
\bar{\xi}_{i}(E)=\frac{\int_{E}^{+\infty} d E_{S}\left[J_{i}\left(E_{S}\right)+4 k_{i}^{2} \int_{E}^{E_{S}} d E^{\prime} \frac{K\left(E^{\prime}\right)}{b_{\text {halo }}\left(E^{\prime}\right)} B_{i}\left(E^{\prime}, E_{S}\right)\right]}{\int_{E}^{+\infty} d E_{S} B_{i}\left(E, E_{S}\right)}
$$

where

$$
J_{i}\left(E_{S}\right)=\frac{1}{h} \int_{0}^{L} d z_{S} \frac{\sinh \left[\frac{S_{i}}{2}\left(L-z_{S}\right)\right]}{\sinh \left[\frac{S_{i} L}{2}\right]} Q_{i}\left(E_{S}, z_{S}\right), \quad k_{i}^{2}=\frac{S_{i}}{8 h} \operatorname{coth}\left(\frac{S_{i} L}{2}\right) .
$$


This procedure consists thus in pinching the halo energy losses inside the disc, hence the name pinching method. Once the effective term $b_{\text {halo }}^{\text {eff }}$ has been computed, it is possible to switch on low-energy effects and to solve Eq. (2.1) with all propagation processes using the usual CrankNicholson algorithm.

The left panel of Fig. 2 represents the flux of secondary positrons computed with the MIN (blue), MED (red) and MAX (vert) sets of propagation parameters. The dotted lines represent the prediction in the high energy approximation (HE) whereas the solid lines are solutions of the full transport equation, computed thanks to the pinching method. The relative error $\left(\Phi_{\mathrm{HE}}^{\mathrm{II}}-\Phi^{\mathrm{II}}\right) / \Phi^{\mathrm{II}}$ arising using the high-energy approximation for secondary positrons is represented in the right panel of Fig. 2. We show that neglecting the low energy effects leads to a sizeable error for the prediction of the positron flux at $10 \mathrm{GeV}$ up to $48 \%$ for the secondary component, and up to $74 \%$ for the DM signal, in contrast to what has been assumed in the literature.

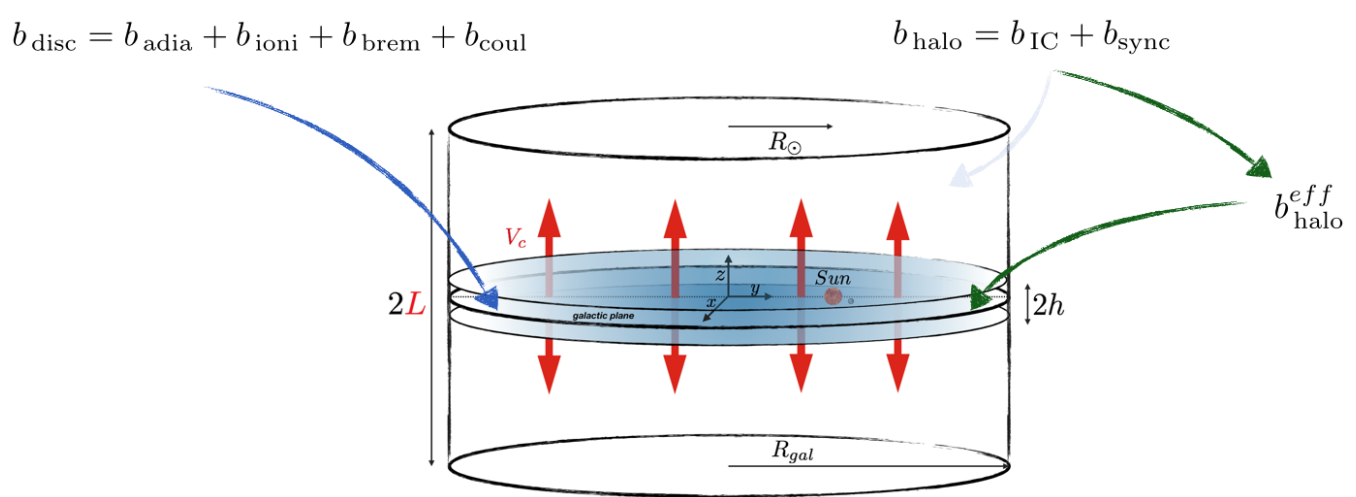

Figure 1: A sketch of the pinching method devised in [4]

\section{Secondary positrons: a probe for the propagation parameters}

For many propagation models compatible with the $\mathrm{B} / \mathrm{C}$ ratio (especially those with a small halo size), pure secondary predictions of the positron flux at the lowest energies (typically below $4 \mathrm{GeV}$ ) are not in deficit but rather in excess with respect to measurements [5]. This observation has been shown to yield a useful complementary constraint on the propagation parameters. Indeed, since the flux of secondary positrons scales as the ratio of the production volume over the diffusion one, leading to $1 / \sqrt{K_{0}}$ dependency, the well-known degeneracy $K_{0} / L$ introduced by secondary-toprimary ratio studies can be lifted.

In the left panel of Fig. 3 we display the AMS-02 positron flux and superimpose the yellow band whose edges correspond to the envelope of the predictions for the secondary positrons computed with the propagation parameters derived fom [3]. The red coloured region represents predictions that overshoot at least one data point more than three standard deviations. As an example, we display in dashed green a model that fulfills our constraint and in dashed red two models that do not respect it. The right panel of Fig. 3 illustrates the allowed propagation models that remain after the selection process. Interestingly, one can see that the positron excess measured by 

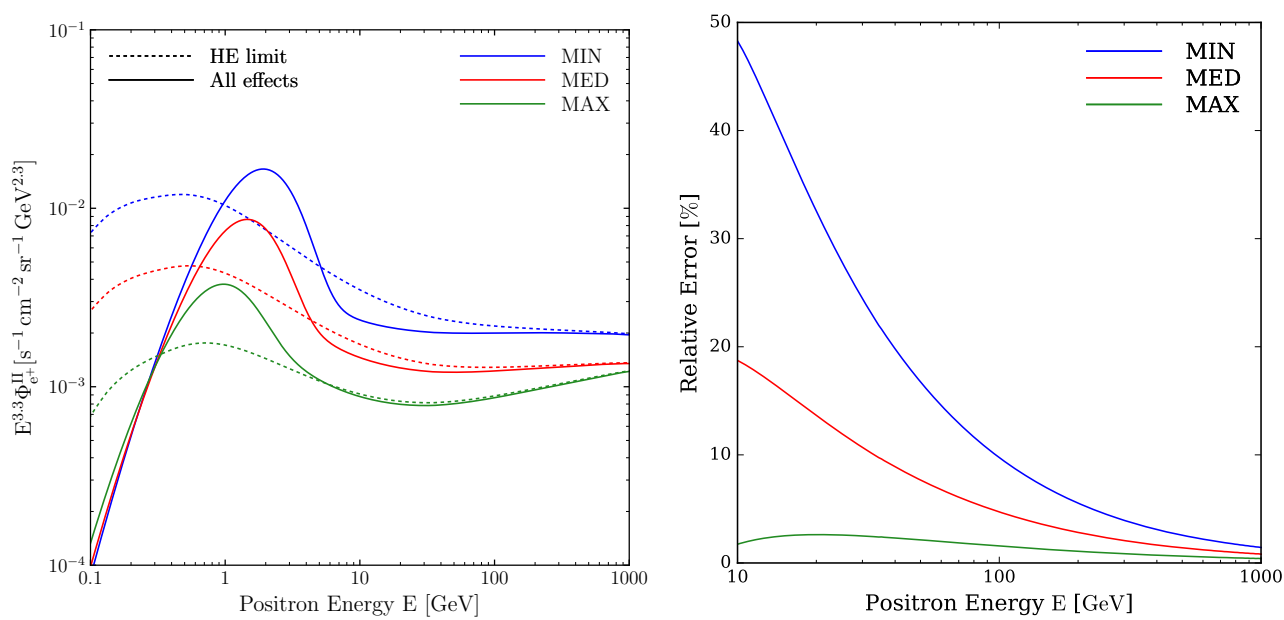

Figure 2: Left: interstellar flux of secondary positrons computed with all propagation effects (solid lines) and with the high-energy approximation ( dotted lines) for the MIN (blue), MED (red), and MAX (green) models. Right: relative error $\left(\Phi_{\mathrm{HE}}^{\mathrm{II}}-\Phi^{\mathrm{II}}\right) / \Phi^{\mathrm{II}}$ above $10 \mathrm{GeV}$ of the high-energy approximation for secondary positrons compared to the exact result.

AMS-02 seems to start already above $2 \mathrm{GeV}$, and not $10 \mathrm{GeV}$ as often advocated. One can see that our new method enables us to drastically reduce the allowed parameter space with respect to former B/C analysis. Pratically, favouring models characterised by i) a large halo size $L$ (ranging from 8.5 to $15 \mathrm{kpc}$ ) together with relatively high $K_{0}$, typically $\geq 0.06 \mathrm{kpc}^{2} \mathrm{Myr}^{-1}$, ii) a slope of the diffusion coefficient $\delta \sim 0.5$. This results are consistent with the trend observed in the antiproton to proton ratio [6] as well as the B/C data recently reported by AMS-02 [7, 8].
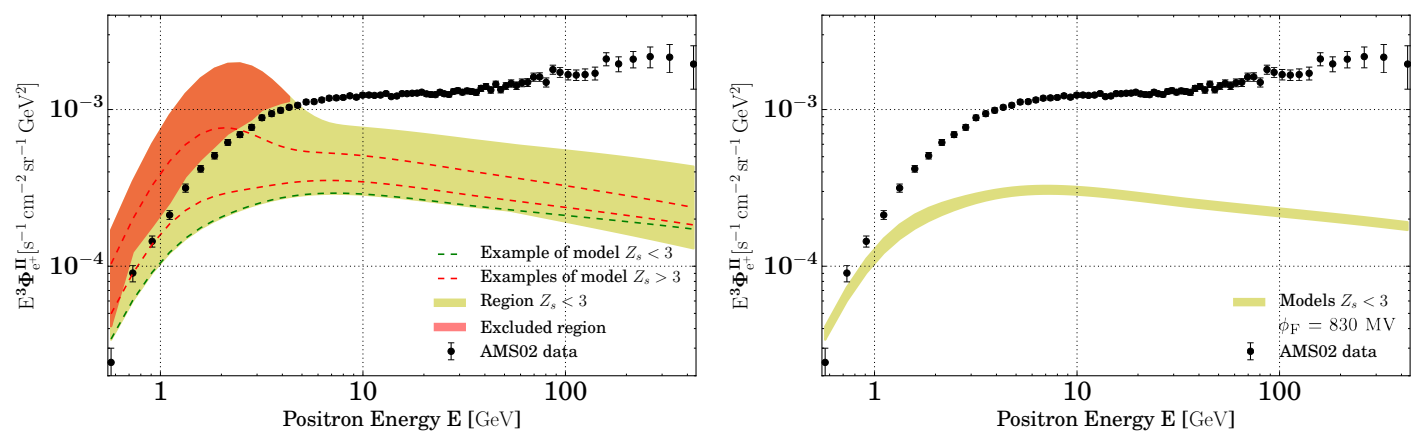

Figure 3: Left: complementarity between the B/C and the positron observables (see text for more details). Right: enveloppe of the positron flux remaining after the AMS-02 constraints. 


\section{Positron excess: the Dark Matter explanation revisited}

The most striking feature of the positron flux data is the high-energy gap with respect to the secondary prediction. Filling this gap with a dark matter component has been the concern of many studies, but semi-analytical methods were always restricted above $10 \mathrm{GeV}$ (see for example [2]). Hereafter, we use the pinching method to compute the positron flux following dark matter annihilation over all the energy range covered by AMS-02 data. Due to the important statistics of data below $10 \mathrm{GeV}$, constraints based only on the quality of the fit become more stringent. We consider two different cases: DM particle annihilating into a general final state composed of quarks, leptons, and bosons, and the case of a leptophilic DM which annihilates into a combination of leptonic channels through a light mediator. In a similar vein as [2], we make no assumptions about the underlying DM model and consider the possibility that DM annihilates into a combination of channels, namely $b \bar{b}, W^{+} W^{-}, e^{+} e^{-}, \mu^{+} \mu^{-}$, and $\tau^{+} \tau^{-}$, with a branching ratio free to vary. The limited choice of these channels relies on the fact that they describe relatively well the various spectrum shape, and avoids introducing too many free parameters. On the other hand, given the high dependence of the spectra on the lepton flavour, we allow non-universal lepton contributions. The DM annihilation spectra of all these channels are calculated using micrOMEGAs_3.6 [10]. Concerning the case of a leptophilic DM, only three branching ratios are introduced as free parameters. They correspond to the three leptonic channels $\left(\phi \phi \rightarrow 2 e^{+} 2 e^{-}, \phi \phi \rightarrow 2 \mu^{+} 2 \mu^{-}, \phi \phi \rightarrow 2 \tau^{+} 2 \tau^{-}\right)$, where $\phi$ is a light scalar mediator. In this case the annihilation spectra are taken from PPPC4DMID [11].

The search for the best fit to the positron data is led in the following way: for twenty DM masses logarithmically distributed from $100 \mathrm{GeV}$ to $1 \mathrm{TeV}$, we perform a fit to the AMS-02 measurements of the positron flux. In the case of the five annihilation channels, the parameter space is of dimension six: two corresponding to $m_{\chi}$ and $\langle\sigma v\rangle$, and four for the branching ratios $b_{i}$ given the constraint $\sum_{i} b_{i}=1$. In the case of the leptophilic DM, the parameter space is of dimension four. To remain conservative, for each propagation model, we perform the fit seven times, varying the Fisk potential in the $3 \sigma$ range [647 MV; $830 \mathrm{MV}$ ] where $724 \mathrm{MV}$ corresponds to the nominal value of the potential[12]. In the direct annihilation case, we find a a global best fit corresponding to a minimal $\chi_{\text {dof }}^{2}=\chi^{2} /$ ndof $=100 / 66=1.5$. It is obtained for a DM mass of $m_{\chi}=264 \mathrm{GeV}$ annihilating into $b \bar{b}, e^{+} e^{-}$, and $\mu^{+} \mu^{-}$with branching ratios of $0.92,0.05$, and 0.03 respectively (the branching ratios for the channels $W^{+} W^{-}$and $\tau^{+} \tau^{-}$are found to be zero). The associated annihilation cross section is $\sim 272$ times larger than the thermal cross section. It means that a peculiar enhancement mechanism is required, as it has been found in many former studies. Similarly, in the leptophilic case, we find a global best fit associated to a $\chi_{\text {dof }}^{2}=1231 / 68=18$. It corresponds to a DM mass $m_{\chi}$ of $183 \mathrm{GeV}$ annihilating into $\phi \phi \rightarrow 2 e^{+} 2 e^{-}$and $\phi \phi \rightarrow 2 \tau^{+} 2 \tau^{-}$with respective branching ratios of 0.09 and 0.91 . The branching ratio of the channel $\phi \phi \rightarrow 2 \mu^{+} 2 \mu^{-}$is chosen as zero by the fit.

Interestingly, values of the minimal $\chi_{\mathrm{dof}}^{2}$ are high, especially in the leptophilic case. Even with numerous degrees of freedom (annihilation channels, DM mass and annihilating cross-section) and considering theoretical uncertainies (propagation, solar modulation) it is not possible to account for the AMS-02 positron data both at low and high energies with the DM scenarios. Somehow, the flatness of the spectrum is such that it is not possible to accommodate the data entirely with a single primary component. Therefore, we find challenging to interpret the excess in terms of pure DM annihilations, since our conservative analysis always leads to low-quality fits of the data. 


\section{MeV Dark Matter: constraints from Voyager I}

$\mathrm{MeV}$ dark matter (DM) particles annihilating or decaying to electron-positron pairs cannot, in principle, be observed via local cosmic-ray (CR) measurements because of the shielding solar magnetic field. Here we take advantage of spacecraft Voyager I's capacity for detecting interstellar CRs since it crossed the heliopause in 2012. This opens up a new avenue to probe DM in the sub$\mathrm{GeV}$ energy/mass range that we exploit here for the first time. From a complete description of the transport of electrons and positrons at low energy using the pinching method, we derive predictions for both the secondary astrophysical background and the pair production mechanisms relevant to $\mathrm{DM}$ annihilation or decay down to the $\mathrm{MeV}$ mass range.

Template predictions for the DM-induced $e^{ \pm}$fluxes are shown in the left panel of Fig. 4, considering WIMPs of $10 \mathrm{MeV}(10 \mathrm{GeV})$ annihilating into $e^{+} e^{-}(b \bar{b})$. In both cases, $e^{+} \mathrm{s}$ and $e^{-}$s share the same injection spectrum and the same propagation history, such that $e^{ \pm}$predictions can be compared to the $e^{+}$data by multiplying the latter by two. We reported our results for propagation models $A$ [13] and $B$ (MAX from [3]), and for the NFW and cored halos. In the weakreacceleration case (model $A$ ), the $e^{ \pm}$flux is suppressed beyond the maximal injected energy set by the dark matter mass $m_{\chi}$, while in the strong-reacceleration case (model $B$ ), low-energy $e^{ \pm} \mathrm{S}$ are reaccelerated beyond $m_{\chi}$. This important feature of the strong-reacceleration regime has, to our knowledge, never been noticed before: DM-induced $e^{ \pm} \mathrm{S}$ could then be observed beyond $m_{\chi}$, which makes the $\mathrm{GeV}$ data also relevant to constrain sub-GeV DM.

We combine the constraints from the Voyager and AMS-02 data to get novel limits covering a very extended $\mathrm{DM}$ particle mass range, from $\mathrm{MeV}$ to $\mathrm{TeV}$. In the $\mathrm{MeV}$ mass range, our limits reach annihilation cross sections of order $\langle\sigma v\rangle \sim 10^{-28} \mathrm{~cm}^{3} / \mathrm{s}$ as shown in the right panel of Fig. 4. An interesting aspect is that these limits barely depend on the details of cosmic-ray propagation in the weak reacceleration case, a configuration which seems to be favoured by the most recent $\mathrm{B} / \mathrm{C}$ data. Though extracted from a completely different and new probe, these bounds have a strength similar to those obtained with the cosmic microwave background (CMB) - they are even more stringent for p-wave annihilation.

\section{Conclusion}

We present a new semi-analytical method to deal with the propagation of Galactic electrons and positrons from $\mathrm{MeV}$ to $\mathrm{TeV}$ energies: the pinching method. Taking advantage of this new tool, we compute the secondary component of positrons and we compare it to the AMS-02 data. This enables us to reduce the parameter space of propagation. Then, we show that the positron excess rises from $\sim 1 \mathrm{GeV}$. We investigate the DM explanation to the positron excess. Interestingly, we find challenging to interpret the excess in terms of pure DM annihilations. Finally, we derive novel constraints on $\mathrm{MeV}$ DM particle using the Voyager I data. These bounds have a strength similar to those obtained with the cosmic microwave background (CMB), even more stringent for $\mathrm{p}$-wave annihilation.

I am grateful to the European Research Council (ERC) under the EU Seventh Framework Program (FP7/20072013)/ERC Starting Grant (agreement n. 278234 NewDark project led by M. Cirelli). 

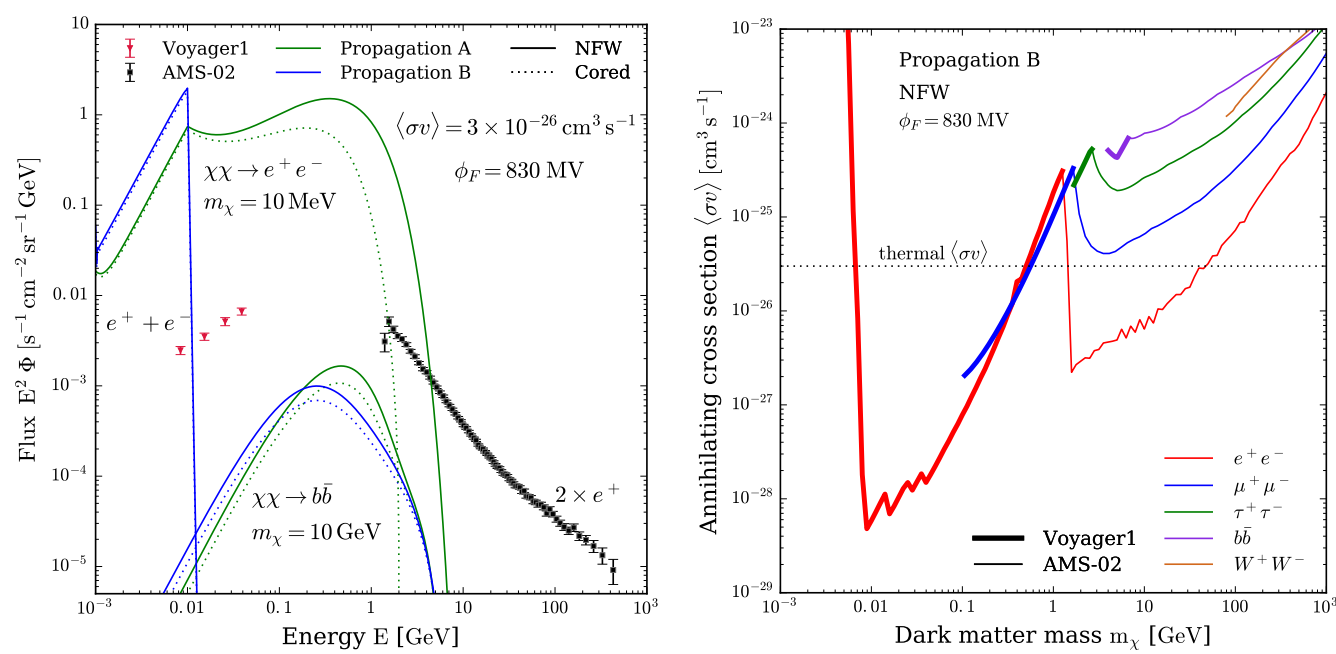

Figure 4: Left: primary electrons and positrons produced by the annihilation of DM particles compared to Voyager I and AMS-02 data. Right: limits for different annihilation final states, assuming configuration $B$-NFW-830 MV.

\section{References}

[1] Aguilar, O. et al., PRL 113, 121102 (2014)

[2] M. Boudaud, S. Aupetit, S. Caroff, A. Putze, G. Belanger, Y. Genolini, C. Goy, V. Poireau, V. Poulin, S. Rosier, P. Salati, L. Tao and M. Vecchi A\&A 575, 67 (2015)

[3] Maurin, D., Donato, F., Taillet, R. and Salati, P. ApJ 555, 585 (2001)

[4] Boudaud, M.,Bueno, E. F., Caroff, S., Genolini, Y., Poulin, V., Poireau, V., Putze, A., Rosier, S., Salati, P. and Vecchi, M., arXiv:1612.03924 (2016)

[5] Lavalle J., Maurin D. and Putze, A., PRD 90, 081301 (2014)

[6] Giesen G., Boudaud M., Genolini Y., Poulin V., Cirelli M., Salati P. and Serpico P. D. JCAP 1509, 09, 023 (2015)

[7] Aguilar, M. et al., PRL 117, 231102 (2016)

[8] Genolini Y., Serpico P.D., Boudaud M., Caroff S., Poulin V., Derome L., Lavalle J., Maurin D., Poireau V., Rosier S., Salati P. and Vecchi M., arXiv:1706.09812 (2017)

[9] Boudaud M., Lavalle J., and S., Salati P., arXiv:1612.07698 (2016)

[10] Bélanger G., Boudjema, F., Pukhov, A. and Semenov, A., Comput.Phys.Commun. 192, 322 (2015)

[11] Cirelli M. et al.JCAP 1106, 018 (2011)

[12] Ghelfi A., Barao Derome L., and Maurin D., A\&A 591, 94 (2016)

[13] Kappl R., Reinert A. and Winkler M. W., JCAP 1510,10, 034 (2015) 